cependant pas le phénomène.

Les indications de la méthode sont :

- essentiellement l'obstruction congénitale du déférent et/ou de l'épididyme par aplasie plus ou moins étendue,

- et l'oblitération post-inflammatoire du tractus épididymo-déférentiel (après MST), non opérable ou après échec d'une anatomose épididymodéférentielle.

(Ces deux indications impliquant bien entendu que la spermatogénèse ait été véréfiée normale et que la tête de l'épididyme ou une partie de celleci au moins soit préservée et accessible.)

\section{CONCLUSION}

1. - Le type de traitement in vitro des spermatozoïdes se révèle d'une grande importance pour la fécondation,

2. - Les premiers résultats positifs ( 2 grossesses gémellaires avec enfants normaux) que nous présentons confirment la possibilité d'éviter le recours au sperme de donneur pour certains de ces couples dont l'azoospermie était jusqu'à considérée comme définitivement incurable, après l'abandon des spermatocèles dont les résultats ont été décevants.

\section{REFERENCES}

1 - Asch R, Ord T, Balmaceda J, Patrizio P, Marel E, Silber $S$. Infertility due to congenital absence of the vas deferens. Results of a new treatment based on epididymal sperm retrieval, in vitro fertilization, and tubal embryo transfer. International Congress of IVF and assisted procreations - Jerusalem - Mai 1989.

2 - Cognat M, Zaroukian A, Bardet J, Czyba J.C, Guérin J.F, Pinatel M.Cl, Lornage J, Regnier-Vigouroux G. Grossesses-Fiv avec sperme épididymaire. A propos de deux succès. Congrès de la S.A.L.F., Sousse 6-8 septembre 1990

3 - Cognat M, Delafontaine D. Utilisation du sperme épididymaire et déférentiel dans un programme de procréation médicalement assistée. 18èmes journées de Fertilité-Orthogénie. Les Nouveautés en Fertilité - Paris 10, 11, 12 novembre 1990.

4 - Guérin J.F, Mathieu C.L, Lornage J, Pinatel M.Cl, and Boulieu D. Improvement of survival and fertilizing capacity of human spermatozoa in a IVF programm by selection on discontinuous Percoll gradients. Human Reproduction. 1989, $4: 798-804$

5 - Orgebin-Crist M.C. Physiologie de l'épididyme et maturation du sperme : état actuels des connaissances. Contraception-Fertilité-Sexualité - 1986, 14 : 487-495.

6- Schoysman R, Bertin G, Van Der Zwalmen P et Segal L. Utilisation du sperme épididymaire dans un programme de FIV; In - Stérilité Masculine et Procréations Médicalement Assistées, Progrès en
Andrologie $n^{\circ} 3$, Y. Englert, J.F Guerin, P. Jouannet eds, Doin, Paris, 1989 : 137-148.

7 - Silber S. Apparent Fertility of Human Spermatozoa from the Caput Epididymis. Andrologia, $1989,10: 263-269$.

8 - Silber S, Balmaceda J, Borrero C, Ord T, Asch R. Pregnancy with sperm aspiration from the proximal head of the epididymis : a new treatment for congenital absence of the vas deferens. Fertility Sterility, 1988, 50 : 525-528.

9 - Temple-Smith P.D, Southwick G.J., Yates C.A, Trounson A.O, De Kretser D.M. Human pregnancy by in vitro fertilization (IVF) using sperm aspirated from the epididymis. Journal of in vitro fertilization and embryo transfert, 1985, $2: 119-122$.

RESUME : L'azoospermie excrétoire, ou plutôt certaines de ses formes anatomocliniques, représente une nouvelle indication de la fécondation in vitro. Deux grossesses obtenues par fécondation in vitro avec spermatozoïdes épididymaires sont l'occasion de faire une revue des applications pratiques de la méthode, et de présenter notre statistique globale reposant sur 15 essais. Mots clés : épididyme, sperme épididymaire, grossesse, FIV, azoospermie excrétoire.

Andrologie, 1991, 1 : 20-21

\title{
NOUVEAUTÉS THÉRAPEUTIQUES EN ANDROLOGIE
}

Une séance organisée par W.B. SCHILL et M. SCHOUMAN le Samedi 30 Novembre à $14 \mathrm{~h}$ va leur être consacrée au cours du congrès de Strasbourg de la Société d'Andrologie de Langue Française.

L'exposé introductif de G. HAIDL (Giessen) précisera l'état actuel des possibilités thérapeutiques en Andrologie.

Puis M. ETCHEVERRY (Bordeaux) fera le point de l'apport de la thermothérapie dans le traitement de l'adénome prostatique. Cette technique récente voudrait éviter ou reculer l'échéance chirurgicale chez certains patients. Tandis que plusieurs fabricants s'emploient à promouvoir activement leur nouveau matériel, l'Association Française d'Urologie a mis en place un programme multicentrique pour l'évaluer objectivement. M. ETCHEVERRY y participe

J. BUVAT terminera la première partie de cette session en faisant le point des injections intra- caverneuses de Prostaglandine El. Cela fait moins de 10 ans qu'ont été découverts les effets spectaculaires de la Papavérine sur l'érection. Seule ou associée à la Phentolamine, cette drogue représente à l'évidence un apport diagnostique et thérapeutique inestimable. La possibilité des complications sérieuses s'est cependant révélée avec le temps. Des alphabloqueurs développés ultérieurement, comme le Moxisylyte, n'ont pas ces inconvénients, mais ne sont pas suffisamment puissants pour tous les cas. La Prostaglandine E1, composé qui pourrait participer à l'érection naturelle, allie puissance et innocuité, mais nous verrons qu'au moins pour l'instant, l'utilisation de cette drogue n'est pas sans inconvénient

Un entracte de $45 \mathrm{~mm}$ sera consacré à une présentation orale des meilleurs posters du congrès.

La $2^{\text {emc }}$ partie de la session sera consacrée à l'infertilité masculine. J. BELAISCH (Paris) a accepté la gageure de faire le point de l'intérêt des traitements médicamenteux dans l'oligospermie idiopathique.

L'exposé de K. DIEDRICH (Bonn) précisera les indications andrologiques des reproductions assistées, et sera une excellente introduction aux 3 communications suivantes:

- micro-injections de spermatozoïdes, traité par J.Ph. WOLF (Paris), qui en a acquis une solide expérience au sein de l'équipe de P. JOUANNET, - prélèvement micro-chirurgical des spermatozoïdes en vue d'insémination, présenté par A.D. WEISKE (STUTTGART),

- enfin Fécondation In Vitro après prélèvement épididymaire ou déférentiel des spermatozoïdes, traité par J.F. GUERIN (Lyon).

Ces 5 communications seront suivies de $30 \mathrm{mn}$ de discussion.

M. SCHOUMAN 